\title{
BIBLIOTHEKARISCH-INFORMATIONSWISSENSCHAFTLICHES WORTSUCHRÄTSEL
}

In der Buchstabensuppe verbergen sich insgesamt 55 bibliothekarische bzw. informationswissenschaftliche Begriffe (waagrecht, senkrecht, diagonal; vorwärts und rückwärts geschrieben; die Wörter können sich überlappen).

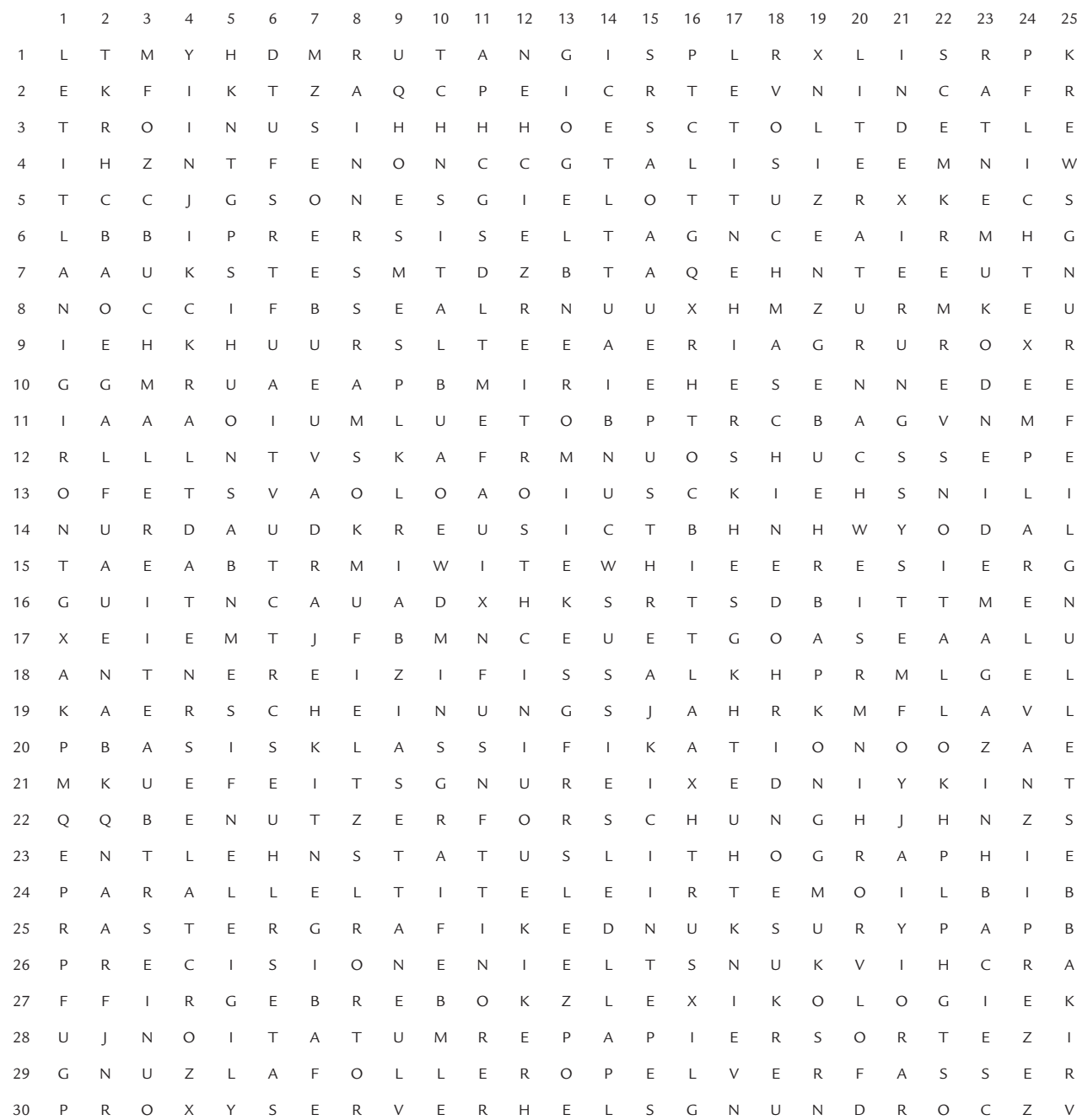

Otto Oberhauser, Wien 
$\left(0 ‘ 6 Z^{\prime} \angle \mathrm{L}\right)$ y

$\left(S^{\prime} \nabla^{\prime} 8 L\right) \exists N I H O S \forall W H O N S$

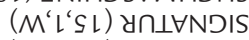

(S'SL'†乙) ZN $\forall \wedge \exists 7 \exists y$

$\left(N^{\prime} L L^{\prime} \angle L\right) 7 \exists \perp \perp N \exists H I \exists Y$

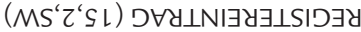

(MS'L'8L) 7า $\forall \supset \exists y$

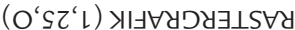

$\left(M S^{\prime} \angle ' 9 L\right) \perp \forall W Y O \exists \perp \forall \forall \cap \circlearrowright$

(O’0ع'L) У

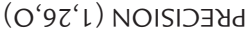

$\left(M S^{\prime} Z^{\prime} L L\right)>>1 I \exists N O H d$

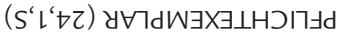

$\left(M^{\prime} 8 z^{\prime} \varepsilon L\right) N O I \perp \forall \perp \cap W Y \exists d$

(ON'0Z' L) NOI $\forall \perp N \exists W \cap>O O \perp N \exists \perp \forall d$

(O‘っでL) ๆ

$\left(M^{\prime} s \tau^{\prime} \nabla \tau\right) \exists O N \cap>S \cap Y \lambda d \forall d$

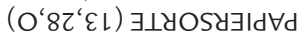

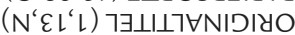

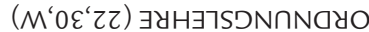

$\left(M^{\prime} \angle Z^{\prime} L L\right) \exists \exists I \mathrm{dD} \exists g y \exists g O$

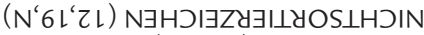

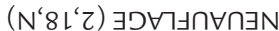

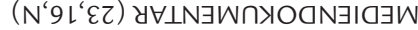

(OS'L'L) yH

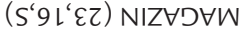

$\left(s^{\prime} \varepsilon^{\prime} 6 L\right)$ y

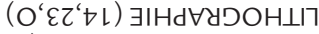

(S'L'0Z) SI $\exists M H O \forall N Y \cap \perp \forall Y \exists \perp I$

(N‘D'sz) >्रУ

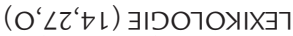

$\left(M^{\prime} 6^{\prime} 0 L\right) \perp-1 S \exists S \exists 7$

$\left(M N^{\prime} 8 L^{\prime} 9 L\right) \perp H D I S \exists \cap \forall 7 \forall \forall S \exists S \exists 7$

(M‘6て'9L) DNกZา $\forall\lrcorner 077 \exists y O d \exists 7$

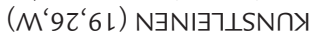

(os'z's) Hวกดช

$\left(M N^{\prime} \varepsilon L^{\prime} \angle L\right) \perp S N \exists I O Y \exists \exists I d O>$

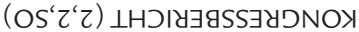

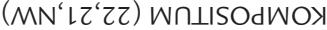

(N`Lて'ZZ) >्रУ $\exists W Y \exists \wedge S N O I \perp \forall 77 O>$

$\left(M^{\prime} 8 L^{\prime} \angle L\right) N \exists \forall \exists \exists I Z I \exists I S S \forall 7>$

(OS'て' )

$\left(M N^{\prime} 8 L^{\prime} Z L\right)$ yO

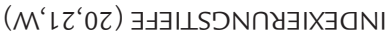

(S‘'เL) W

(O‘6L'ع) yH

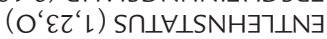

$\left(S^{\prime} 9^{\prime} \varepsilon\right)$ 状

(OS‘9‘z) $\exists$ HI

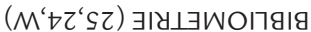

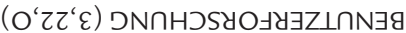

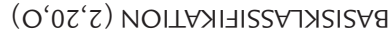

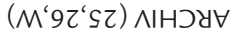

(S‘レ゙ฑ) N $\exists \perp \forall \square \perp 7 \forall$

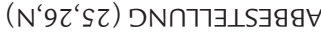

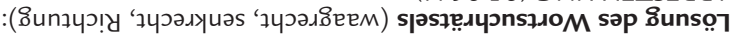

